\title{
Displacement Measurement for High Speed Tribological Measurement Using Oscillating Optical Fiber Probe"
}

\author{
Shintaro ITOH $^{* *}$, Koki IMAI ${ }^{* *}$, Kenji FUKUZAWA $^{* *}$, Yuya HAMAMOTO ${ }^{* *}$ \\ and Hedong ZHANG ${ }^{* * *}$ \\ **Department of Micro-Nano Systems Engineering, Graduate School of Engineering, Nagoya University, \\ Furo-cho, Chikusa-ku, Nagoya, 464-8603, JAPAN \\ E-mail: s_itoh@nuem.nagoya-u.ac.jp \\ ***Department of Complex Systems Science, Graduate School of Information Science, Nagoya \\ University, \\ Furo-cho, Chikusa-ku, Nagoya, 464-8601, JAPAN
}

\begin{abstract}
Lubrication of the head disk interface (HDI) of a hard disk drive is achieved by coating a magnetic disk with a liquid lubricant film of monolayer thickness. It is important to clarify the tribological properties of the lubricant film for optimal lubrication of the HDI. In our previous study, we developed a highly sensitive method to measure shear force, which we called the "fiber wobbling method (FWM)." This method enables us to measure the viscoelastic properties of molecularly thin lubricant films. However, the shearing speeds were less than a few hundred $\mu \mathrm{m} / \mathrm{s}$, which is much slower than expected for the HDI. In this study, we developed a novel technique to measure displacement with a wide measuring range and high sensitivity, which will be essential for high speed tribological measurement using FWM.
\end{abstract}

Key words: Displacement Measurement, Tribological Measurement, Head Disk Interface, Lubrication, Optical Fiber

\section{Introduction}

An understanding of the tribological properties of a molecularly thin lubricant film is necessary to optimize the lubrication at the head-disk interface (HDI) of high-recording-density hard disk drives. Characteristic conditions of lubricant shearing at the HDI are confinement of lubricant molecules in nanometer-sized gap widths and high shearing speeds around 1 to $10 \mathrm{~m} / \mathrm{s}$. In our previous study, we developed a highly sensitive shear force measuring method, which we called the "fiber wobbling method (FWM)" (1)(2). In this method, we use an optical fiber with a spherical-shaped tip as a shearing probe (Fig. 1). The optical fiber probe is oscillated sinusoidally by a piezo actuator, and a sample is sheared by its end. The shear forces acting on the probe tip are detected by the deflection of the fiber. Using our method, we revealed that the molecularly thin lubricants showed characteristic viscoelastic properties such as enhanced viscosity and elasticity. However, in the previous experiments, shearing speeds were less than a few hundred $\mu \mathrm{m} / \mathrm{s}$, and they were much smaller than those in the HDI. Therefore, we have tried to utilize the resonant oscillation of the optical fiber probe to achieve higher shearing speeds. Since the shearing speed was determined by the product of the oscillation amplitude and the frequency in FWM, higher shearing speeds must be achieved by enhancing the probe amplitude. In the

${ }^{*}$ Received 1 Aug., 2009 (No. 09-0410) [DOI: 10.1299/jamdsm.4.2]

Copyright $\odot 2010$ by JSME 


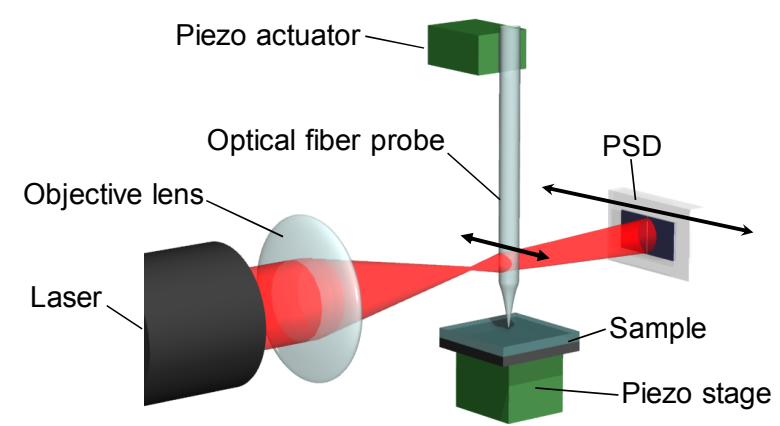

Fig. 1 Schematic diagram of fiber wobbling method. The piezo actuator is used to oscillate the optical fiber probe sinusoidally. The sample is sheared by the probe tip, and the probe's deflection is optically detected. The shearing gaps between the probe tip and the substrate are controlled by using the piezo stage.

resonant oscillation, the probe amplitude $A_{\text {probe }}$ can be determined by the following equation,

$$
A_{\text {probe }}=Q \cdot A_{\text {piezo }}
$$

Here, $A_{\text {piezo }}$ is the amplitude of the piezo actuator that oscillates the probe, and $Q$ is the $\mathrm{Q}$ factor of the probe. Equation (1) indicates that the probe amplitude was enhanced by the Q factor in the resonant oscillation. Usually, the optical fiber used in the FWM has a Q factor of around 1000 and a resonant frequency of 1-20 kHz. The amplitude of the piezo actuator, $A_{\text {piezo }}$, is in the range of a few to a few hundred $\mathrm{nm}$. Therefore, the maximum amplitudes that can be achieved by using the resonant oscillation should be in the range of a few to tens of $\mu \mathrm{m}$, which corresponds to the shearing speeds of 1 to $10 \mathrm{~m} / \mathrm{s}$. According to the above estimation, although the higher shearing speed of up to $10 \mathrm{~m} / \mathrm{s}$ is expected to be achieved, it is essential to develop a displacement measurement that can measure the amplitude of a few tens of $\mu \mathrm{m}$. In our previous study, the displacement of the probe was measured by focusing the laser beam by using the fiber as a cylindrical lens and detecting the movement of the focused laser spot irradiated on the position sensitive detector (PSD $)^{(3)}$. This method provides high sensitivity of around $0.01 \mathrm{~nm}$. However, its measuring range was less than a few $\mu \mathrm{m}$, since the spherical aberration of the fiber, which is used as the lens, caused blurring of the laser spot. This means that the displacement measurement used in the previous study cannot be applied to the high speed shearing with the FWM. The displacement measurement should also be sufficiently sensitive to detect the probe deflection caused by the shearing of the lubricant film. In this study, we have developed a novel method of displacement measurement that provides a wide measuring range and high sensitivity.

\section{Concept and theoretical analyses of the novel displacement measurement method}

Figure 2 is a schematic illustration of the displacement measurement technique we have developed in this study. A laser beam is irradiated near the probe tip, and the shadow of the probe is formed on the position sensitive detector (PSD). The PSD is a photo detector that outputs a current signal that is proportional to the position of the center of the irradiated laser intensity. To create a clear shadow on the PSD, the fiber probe was metalized except at its end (see inset of Fig. 2). If the probe moves, its shadow also moves. Therefore, the displacement of the probe can be measured by detecting the changes in the irradiation area on the PSD. The advantage of this method is that the probe displacement can be measured 


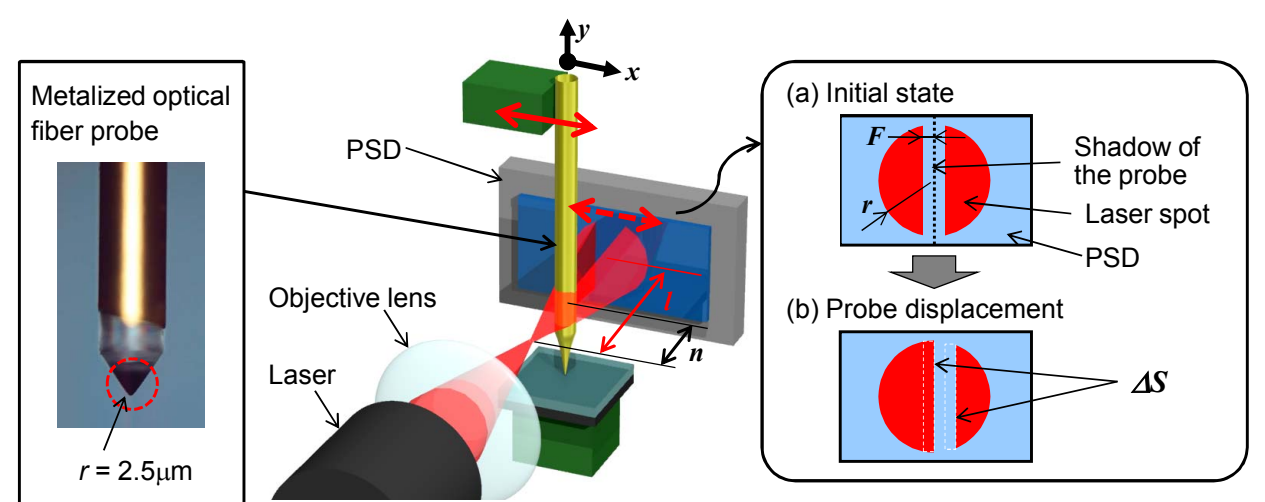

Fig. 2 Schematic of displacement measurement utilizing the detection of the probe shadow. Insets show a micrograph of the metalized probe and the schematics explaining the movement of the shadow on the position sensitive detector (PSD).

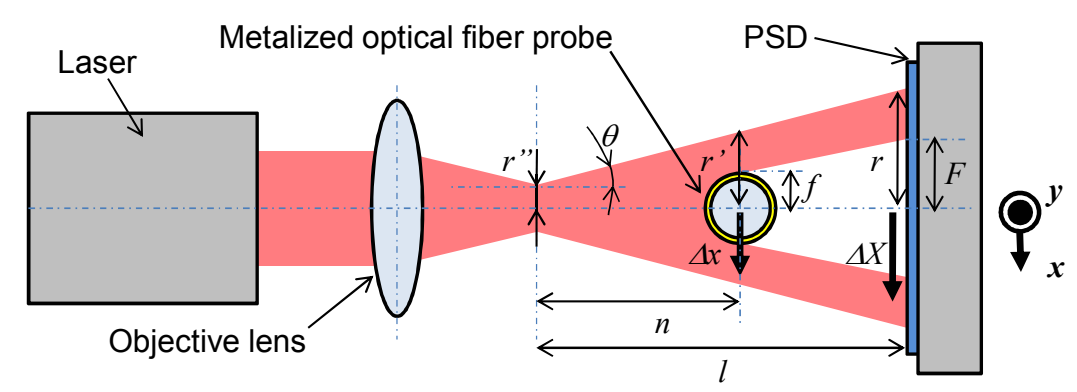

Fig. 3 Optical system for displacement measurement viewed from the axial direction of the probe.

on the condition that the displacement is less than the width of the laser beam. In addition, the width of the laser beam can be increased, in contrast to the probe displacement, if the distance between the probe and the focal point of the laser beam, which is represented as $n$ in Fig. 2, is set larger.

As shown in Fig. 2, we assumed that the circular laser spot with radius $r$ was irradiated on the PSD, and the shadow of the fiber was formed inside the laser spot as a rectangular shape with width $2 F$. Figure 3 shows the optical system viewed from the probe's axial direction. We defined the direction of the probe displacement as the $x$ direction. The $y$ direction, which is perpendicular to the $x$ direction, was set in the plane of PSD's detection area as shown in Fig. 3. Origins of these axes were at the center of the PSD. For the initial state, we assumed both the laser spot and the shadow were at the center of the PSD. In this condition, the current signal from the PSD is zero since the distribution of the irradiated laser intensity is balanced in the PSD's detection area. If the shadow moves by $\Delta X$ in the $x$ direction as shown in Fig. 2(b), the irradiated area increases by $\Delta S$ on the left half of the detection area. On the contrary, it decreases by $\Delta S$ on the right half of the plane. This leads to the shift in center position of laser spot's intensity distribution. If the shift of the center position is denoted by $\Delta X_{c}$, the current signal $I_{s}$ obtained from the PSD is represented as,

$$
I_{s}=\frac{2 I_{0} \Delta X_{c}}{L_{X}}
$$

Here, $I_{0}$ and $L_{X}$ are total photoexcited current and the resistance length, respectively. The resistance length is equal to the length of the PSD's detection area in the $x$ direction. Generally the intensity distribution of the circular laser spot with radius $r$ is represented as, 


$$
d(x, y)=\frac{2 P}{\pi r^{2}} \exp \left(\frac{-2\left(x^{2}+y^{2}\right)}{r^{2}}\right)
$$

where $P$ denotes the total laser intensity. When the probe's shadow with width $F$ moves by $\Delta X$, the shift of the center position $\Delta X_{c}$ is written as,

$$
\Delta X_{c}=\frac{\int_{\Delta X+F}^{r} \int_{-r}^{r} x \cdot d(x, y) \mathrm{dydx}+\int_{-r}^{\Delta X-F} \int_{-r}^{r} x \cdot d(x, y) \mathrm{dydx}}{\int_{\Delta X+F}^{r} \int_{-r}^{r} d(x, y) \mathrm{dydx}+\int_{-r}^{\Delta X-F} \int_{-r}^{r} d(x, y) \mathrm{dydx}}
$$

The total current $I_{0}$ is

$$
I_{0}=u\left(\int_{\Delta X+F}^{r} \int_{-r}^{r} d(x, y) \mathrm{dydx}+\int_{-r}^{\Delta X-F} \int_{-r}^{r} d(x, y) \mathrm{dydx}\right)
$$

where $u$ represents the sensitivity of the PSD. Based on the optical system shown in Fig. 3, following Eqs. (6) to (8) represents relationship between the shadow's displacement $\Delta X$ and the probe's displacement $\Delta x$, that between the width of the shadow $F$ and the probe's radius $f$, and that between the radius of laser spot on the PSD, which is $r$, and that at the probe position $r$ ', respectively.

$$
\begin{aligned}
& \Delta X=\frac{l}{n} \Delta x \\
& F=\frac{l}{n} f \\
& r=\frac{l}{n} r^{\prime}
\end{aligned}
$$

Here, the distance from the focal point of the objective lens to the PSD is represented by $l$, and that distance to the probe is $n$. The radius of laser spot at the probe position $r$ ' is determined by divergence angle of the laser beam $\theta$, the distance $n$, and the radius of the laser spot at the focal point $r^{\prime \prime}$. Therefore it is written as,

$$
r^{\prime}=r^{\prime \prime}+n \tan \theta
$$

Assuming the diffraction limit spot, the radius $r^{\prime \prime}$ is written as,

$$
r^{\prime \prime}=\frac{0.61 \lambda}{N}
$$

where $\lambda$ is the wavelength of the laser and $N$ is the numerical aperture of the objective lens. The divergence angle of the laser beam $\theta$ is

$$
\theta=\sin ^{-1} N
$$

Substituting Eqs. (10) and (11) into Eq. (9), the radius $r^{\prime}$ is written as, 


$$
r^{\prime}=\frac{n N}{\sqrt{1-N^{2}}}+\frac{0.61 \lambda}{N}
$$

Using Eqs. (2) to (8), the current signal $I_{s}$ is obtained as,

$$
I_{s}=-\frac{l P u r^{\prime} \cdot \exp \left(-2\left(\Delta x^{2}+f^{2}\right) / r^{\prime 2}\right) \cdot\left(-1+\exp \left(8 \Delta x f / r^{\prime 2}\right)\right) \cdot \operatorname{Erf}(\sqrt{2})}{\sqrt{2 \pi} n L_{X}}
$$

Here, Erf represents an error function. The following Eq. (14) is the linearized function of Eq. (13), which is obtained by Taylor expansion in a neighborhood of $x=0$ in order to discuss the linearity of the displacement measurement.

$$
\bar{I}_{s}=-\frac{4 \sqrt{2 / \pi} f l P u \cdot \exp \left(-2 f^{2} / r^{\prime 2}\right) \cdot E r f(\sqrt{2}) \Delta x}{r^{\prime} n L_{X}}
$$

Substituting typical values of $l=2 \mathrm{~cm}, P=3 \mathrm{~mW}, \quad u=0.4 \mathrm{~A} / \mathrm{W}, f=50 \mu \mathrm{m}$, $L_{X}=6 \mathrm{~mm}, N=0.2$, and $\lambda=635 \mathrm{~nm}$ to Eqs. (12) to (14), the relationship between the probe displacement $\Delta x$ and the current signal from the PSD was obtained as shown in Fig. 4. Distance $n$ was varied from $0.5 \mathrm{~mm}$ to $6.0 \mathrm{~mm}$. Solid lines are the calculated results of $I_{s}$ and dashed lines are the results of the linearized function $\bar{I}_{s}$. Since it is obvious that the relationship between $\Delta x$ and $I_{s}$ is origin symmetry, Fig. 4 shows only the results of positive displacement $\Delta x$. When the probe displacement $\Delta x$ is in the positive direction, movement of the probe's shadow is also in the positive direction. Therefore the shift of the center position of the laser intensity is in the negative direction, which leads to the increase of the negative current signal $I_{s}$. Since $\Delta x$ and $I_{s}$ showed nonlinear relationship as shown in Fig. 4, displacement range where $I_{s}$ can be approximated by $\bar{I}_{s}$ is the applicable measuring range in the actual displacement measurement. To verify the applicable displacement range, we calculated the approximation errors between $I_{s}$ and $\bar{I}_{s}$ using the following equation.

$$
\operatorname{Dev}=\frac{\bar{I}_{s}-I_{s}}{I_{s}}
$$

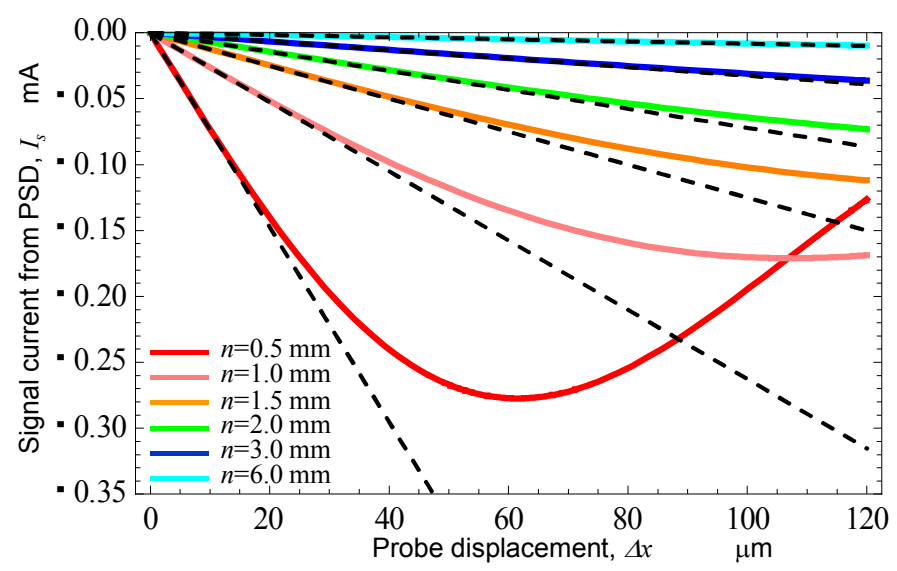

Fig. 4 Relationship between the current signal and the displacement of the probe $\Delta x$. Solid lines represent exact values of current signal $I_{s}$ calculated using Eq. (13) and dashed lines are the linearly-approximated values calculated by Eq. (14). Distance $n$ was varied from $0.5 \mathrm{~mm}$ to $6.0 \mathrm{~mm}$. 


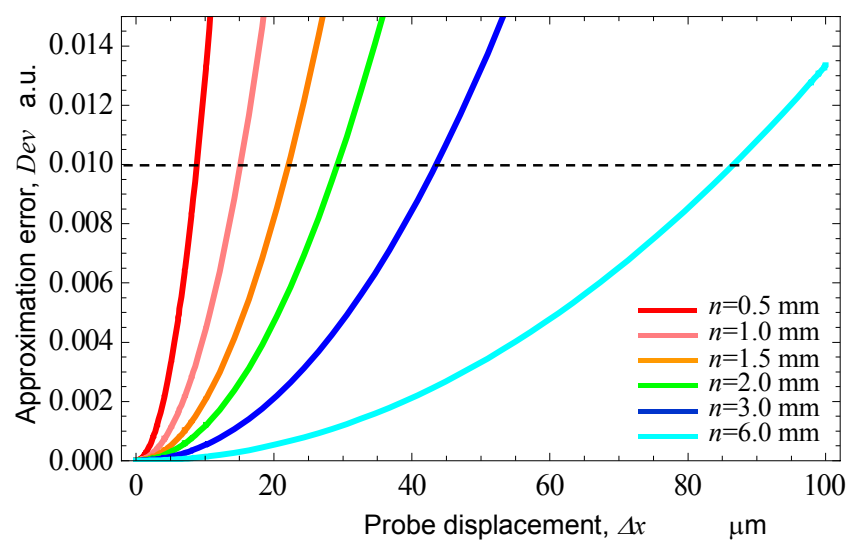

Fig. 5 Approximation errors between $I_{s}$ and $\bar{I}_{s}$ calculated by using Eq. (15) at different distance $n$.

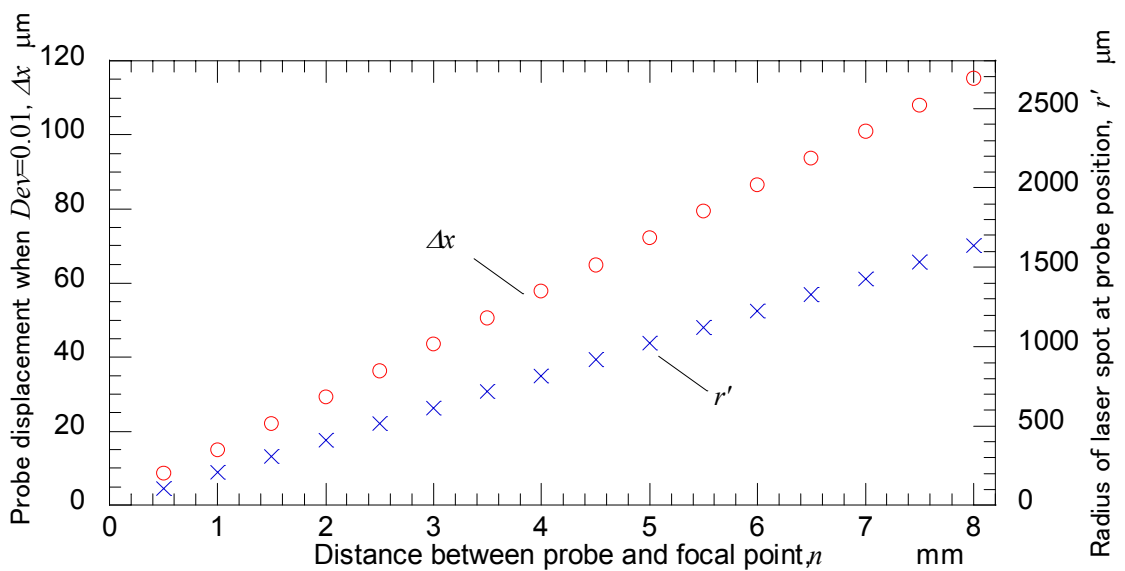

Fig. 6 Effect of distance $n$ on the probe displacement that satisfies $D e v=0.01$ and on the radius of laser spot at the probe position.

Figure 5 plots the calculated Dev against $\Delta x$ with different distances of $n$ ranging from $0.5 \mathrm{~mm}$ to $6.0 \mathrm{~mm}$. As shown in Fig. 5, Dev increases monotonically along with the increase of $\Delta x$. To reveal the effect of distance $n$ on the applicable displacement range, we numerically calculated $\Delta x$ that satisfies $D e v=0.01$ and plotted against $n$ as shown in Fig. 6. Figure 6 also shows the radius of the laser spot at the probe position. From the calculated results in Fig. 6, it is considered that the applicable displacement range can be linearly increased with the increase of distance $n$. In addition, the radius of laser spot $r^{\prime}$ is expected to be larger than a few dozen times of the probe's radius, which is around $50 \mu \mathrm{m}$, to achieve the displacement measurement over a few tens of $\mu \mathrm{m}$.

The theoretical signal-to-noise ratio (SNR) of the displacement measurement was derived as follows. As a displacement signal, we used the linearized current signal that was represented by Eq. (14). As a noise current, we considered only the shot noise. This is because the dark current noise must be negligibly small compared with the shot noise since the irradiated laser intensity was sufficiently large. Therefore, the noise current is expressed as,

$$
I_{n}=\sqrt{2 P b q u}
$$

where $b$ and $q$ are the bandwidth of the detection circuit and the electron charge, respectively. From Eqs. (14) and (16), the theoretical SNR is written as, 


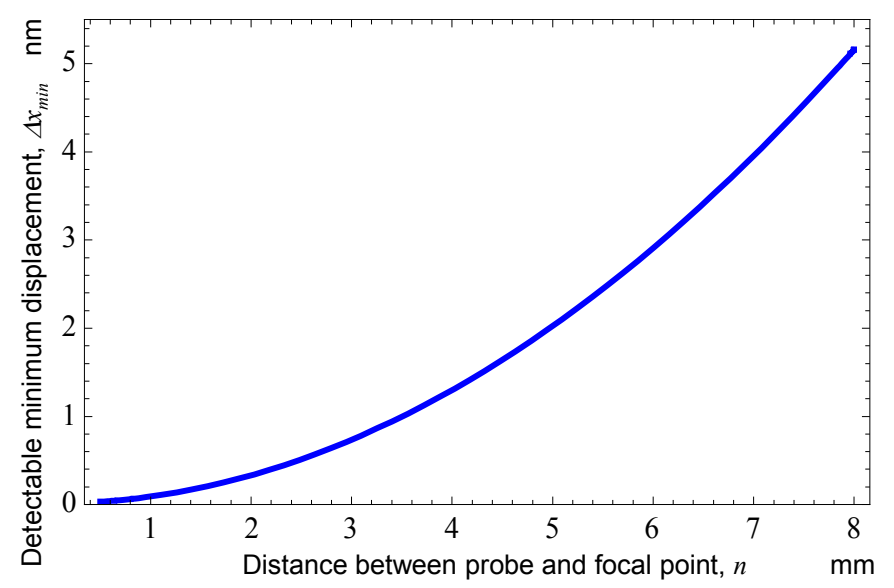

Fig. 7 Relationship between detectable minimum displacement and the distance $n$.

$$
S N R=\frac{\left|I_{s}\right|}{I_{n}}=\frac{4 f l P u \cdot \exp \left(-2 f^{2} / r^{\prime 2}\right) \cdot \operatorname{Erf}(\sqrt{2}) \Delta x}{\sqrt{\pi} r^{\prime} n L_{X} \sqrt{P b q u}}
$$

The detectable minimum displacement $\Delta x_{\min }$ can be determined by the displacement when $S N R=1$, which can be written as,

$$
\Delta x_{\min }=\frac{\sqrt{\pi} r^{\prime} n L_{X} \sqrt{P b q u} \cdot \exp \left(2 f^{2} / r^{\prime 2}\right)}{4 f l P u \cdot \operatorname{Erf}(\sqrt{2})}
$$

Substituting typical values of $l=2 \mathrm{~cm}, P=3 \mathrm{~mW}, \quad u=0.4 \mathrm{~A} / \mathrm{W}, f=50 \mu \mathrm{m}$, $L_{X}=6 \mathrm{~mm}, N=0.2, \lambda=635 \mathrm{~nm}, b=150 \mathrm{~Hz}, q=1.6 \times 10^{-19} \mathrm{C}$ to Eqs. (12) and (18), relationship between $\Delta x_{\min }$ and the distance $n$ was obtained as shown in Fig. 7. From the calculated results of $\Delta x_{\min }$, we considered the displacement measurement had enough sensitivity to detect the probe oscillation at the shearing experiment.

Calculated results shown in Fig. 6 indicate that the distance $n$ must be longer to achieve the wider measuring range. However, due to results shown in Fig. 7, the longer $n$ degrades the SNR and leads to reduced sensitivity. Therefore, the optimum values of $n$ should be adjusted considering the measuring range and the sensitivity required for the tribological measurement with FWM.

\section{Experiment}

\subsection{Experimental setup}

We conducted an experiment to verify our new displacement measurement method. Figure 8 is a schematic diagram of the experimental setup we constructed. The piezo actuator was oscillated by the amplified oscillation signal from the function generator. A laser diode with a maximum power of $3 \mathrm{~mW}$ was used as the light source. Wavelength of the laser was $635 \mathrm{~nm}$. For the PSD, we used a one-dimensional PSD with a detection area of $1 \times 6 \mathrm{~mm}$. The diameter of the optical fiber probe was around $100 \mu \mathrm{m}$, and its tip had a curvature radius of $2.5 \mu \mathrm{m}$. The length of the probe was $5 \mathrm{~mm}$. The displacement signal from the PSD, which was the current signal, was converted to voltage and amplified at the signal processor. The amplitude and phase of the amplified displacement signal was detected by using the lock-in amplifier. The oscillation signal from the function generator was used as the reference signal in this synchronized detection. The noise component was 


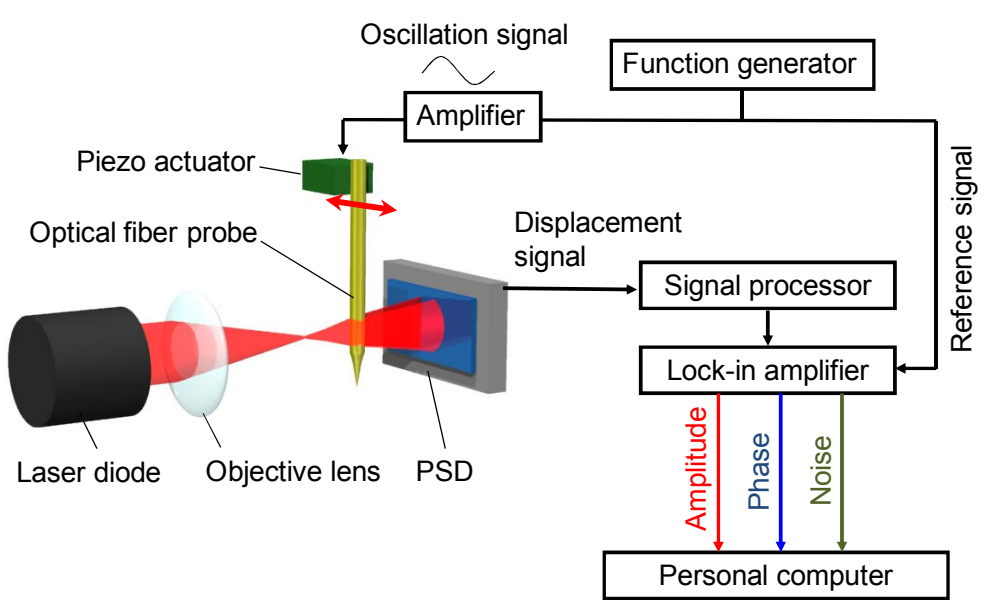

Fig.8 Schematic diagram of experimental setup.

also measured to evaluate the SNR of the method. These measured values were recorded in the personal computer. Although the recorded phase is essential for the viscoelastic evaluation, it was not used to verify the technique of displacement measurement in this study.

\subsection{Experimental procedure}

We carried out a calibration of the measurement method. The calibration curve was obtained by pushing the probe tip using the sharp needle attached on the micrometer stage. Since the feed distance of the micrometer stage was equal to the displacement of the probe tip, we were able to obtain the relationship between the probe displacement and the displacement signal from the PSD. During this calibration, the synchronous detection using the lock-in amplifier could not be used because the probe was not oscillated. Therefore, the displacement signal amplified by the signal processor was recorded directly in the personal computer.

Next, we experimentally verified the detectable minimum displacement $\Delta x_{\min }$ of the measurement. In this experiment, the probe was oscillated sinusoidally. The oscillation amplitude was decreased linearly by decreasing the driving voltage of the oscillating piezo actuator. In the meantime, both the displacement signal, which was the probe amplitude, and the noise signal were measured simultaneously by using the lock-in amplifier whose bandwidth was set at $150 \mathrm{~Hz}$. Figure 9 plots the typical result, which shows the relationship between the driving voltage for the piezo actuator and the measured displacement signal and its noise component. The horizontal axis is the driving voltage of the piezo actuator. The green plots are the displacement signal, and the red plots are the noise component. The blue line represents the linearly approximated line to the displacement signal. As shown in the figure, the detectable minimum displacement was determined as the probe amplitude estimated from the driving voltage at the point where the approximated line intersected with the noise level.

The measuring range of our method was determined experimentally by measuring the detectable maximum displacement. In this measurement, the probe was oscillated sinusoidally, and the oscillation frequency was swept in a certain frequency range that included the probe's resonant frequency at its center. The driving voltage of the piezo actuator was constant $\left(90 \mathrm{~V}_{\mathrm{p}-\mathrm{p}}\right)$. If the measuring range was wide enough compared with the probe amplitude, the single resonance peak of the probe could be detected. However, if the probe amplitude became larger than the dynamic range, the displacement signal discontinuously dropped at a certain amplitude level, as shown in Fig. 10. The reason for this decay might be that the oscillation signal from the PSD could not be synchronously 


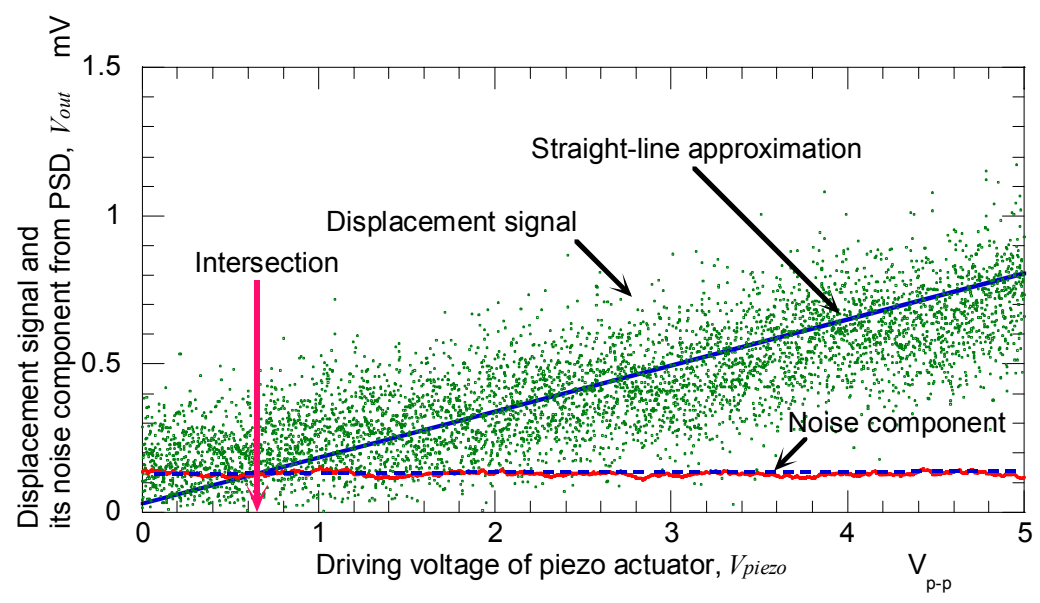

Fig. 9 Relationship between the linearly reduced driving voltage of the piezo actuator and the output signal from the PSD. Green plots represent the signal components, and red plots are its noise components. The point at the intersection of the approximated line and the noise level gives the minimum voltage required to drive the probe at a detectable minimum amplitude.

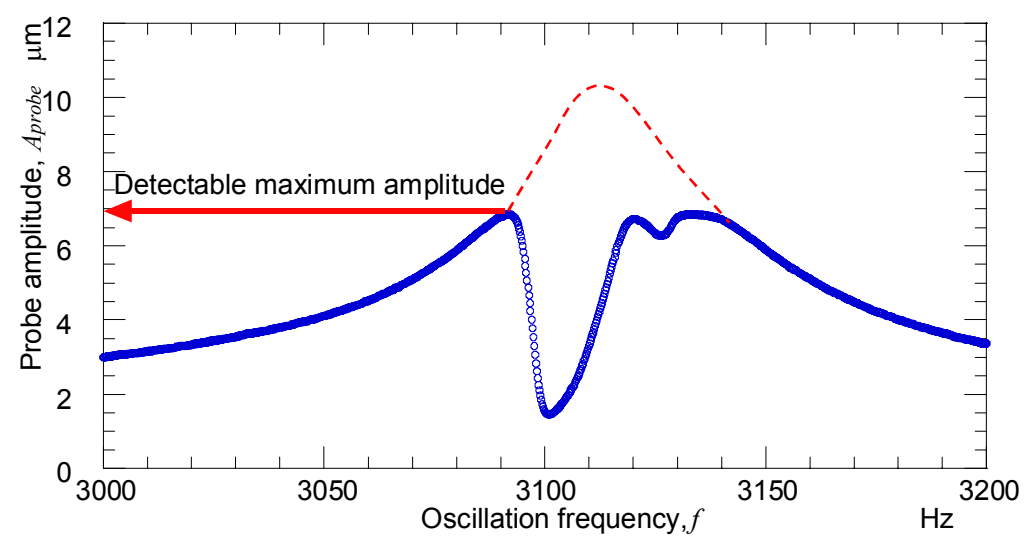

Fig. 10 Detectable maximum displacement determined by measuring the resonance curve of the optical fiber probe.

detected with the lock-in amplifier since its sinusoidal waveform was distorted due to the nonlinear-relationship between the probe displacement and the current signal as shown in Fig. 4. Therefore, we defined the probe amplitude measured just before the decay occurred as the detectable maximum displacement $\Delta x_{\max }$.

Based on the theoretical consideration in $\S 2$ (Eq. (18)), the detectable minimum displacement $\Delta x_{\min }$ depends on the distance $n$. A smaller distance $n$ leads to smaller values of $\Delta x_{\min }$, which means higher sensitivity. In addition, as already mentioned, the detectable maximum displacement $\Delta x_{\max }$ can be increased due to the larger $n$ values in principle. We experimentally measured $\Delta x_{\min }$ and $\Delta x_{\max }$ at different $n$ distances to verify the concept and the performance of our method. Distance $n$ was fed from the initial position of the probe by $1.0-\mathrm{mm}$ steps.

\subsection{Results and discussion}

The calibration curve of the displacement measurement obtained by the procedure explained above is plotted in Figs. 11 and 12. Figure 11 is the results measured on the condition that the detectable maximum amplitude was $9.2 \mu \mathrm{m}$. In Fig. 11, the triangles 


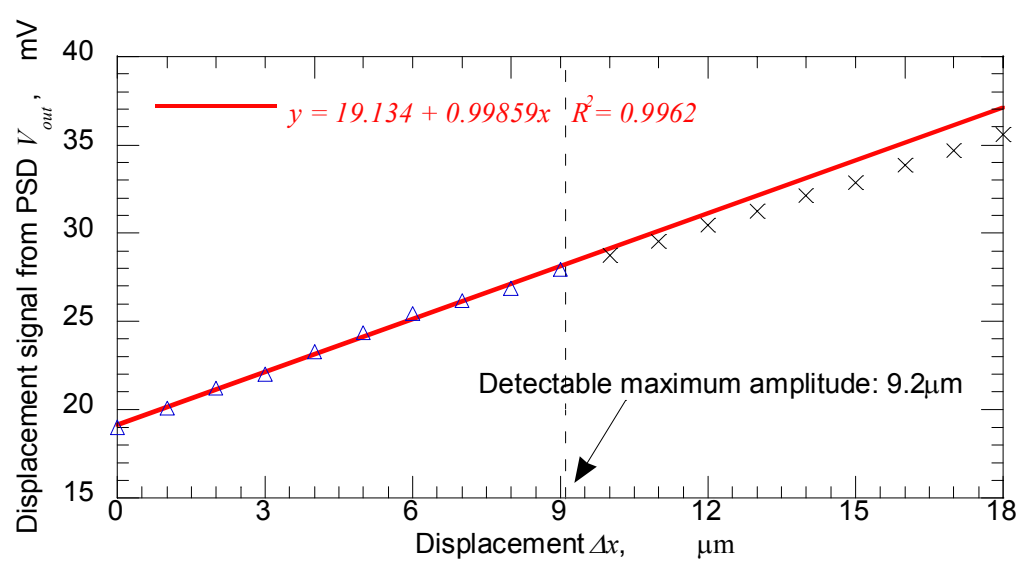

Fig. 11 Calibration curve of displacement measurement obtained with the optical alignment that had the detectable maximum amplitude of $9.2 \mu \mathrm{m}$.

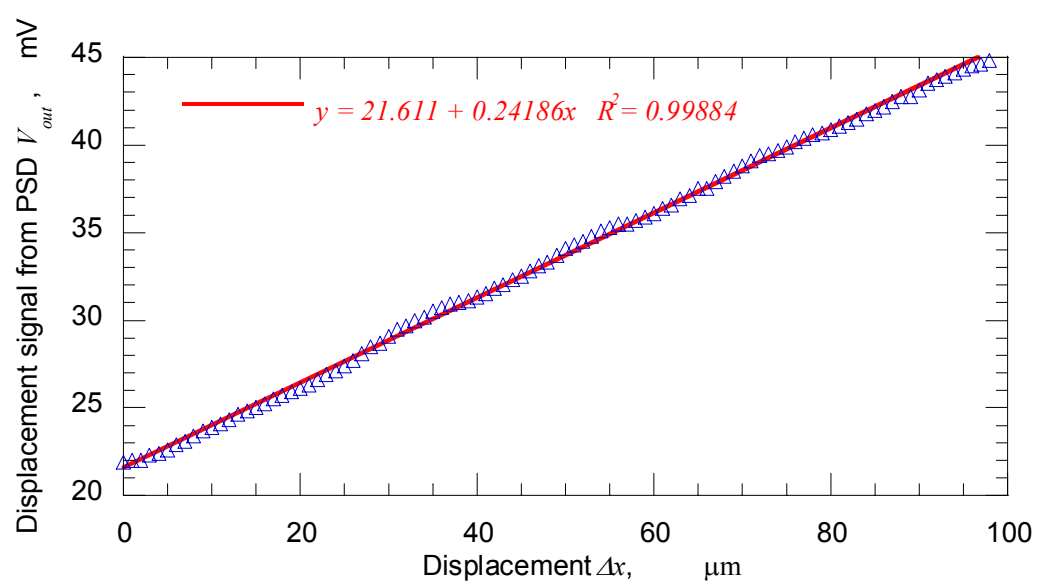

Fig. 12 Calibration curve of displacement measurement obtained on the condition that the maximum displacement of $100 \mu \mathrm{m}$ can be measured.

were the displacement signals measured within the detectable amplitude range and the crosses were those outside. On the other hand, results shown in Fig. 12 were obtained when the distance $n$ was long enough to detect the largest probe displacement, which was $100 \mu \mathrm{m}$. The red line in Fig. 11 and 12 shows the results of straight-line approximation using the least-squares method. In Fig. 11, the approximation was applied only to triangle plots. The determination coefficient derived from the approximate calculation was around 0.99 in both cases. This result indicates that our method had high linearity within the detectable amplitude range. Especially, Fig. 12 shows that a linear relationship between the displacement signal and the probe displacement was achieved up to the maximum displacement of $100 \mu \mathrm{m}$. The linearity over $100 \mu \mathrm{m}$ could not be evaluated due to the limit of the feed distance of the micrometer stage.

With our experimental setup, it is difficult to determine precise values of the distance $n$ and those of laser spot's radius at the probe position, which is represented as $r$ '. However, they can be estimated from the theoretical relationship expressed as Eq. (15). Linearity up to $9.2 \mu \mathrm{m}$ must be achieved by setting distance $n$ larger than $538 \mu \mathrm{m}$ that corresponds to the laser spot radius $r$ ' of larger than $112 \mu \mathrm{m}$, which is about twice as large as the probe radius of $50 \mu \mathrm{m}$. In the same manner, the linearity up to $100 \mu \mathrm{m}$ was considered to be achieved at $n>6931 \mu \mathrm{m}$ and $r^{\prime}>1417 \mu \mathrm{m}$.

Figure 13 plots the experimental results that show the effect of different values of distance $n$ on the detectable minimum displacement $\Delta x_{\min }$. Although the relative 


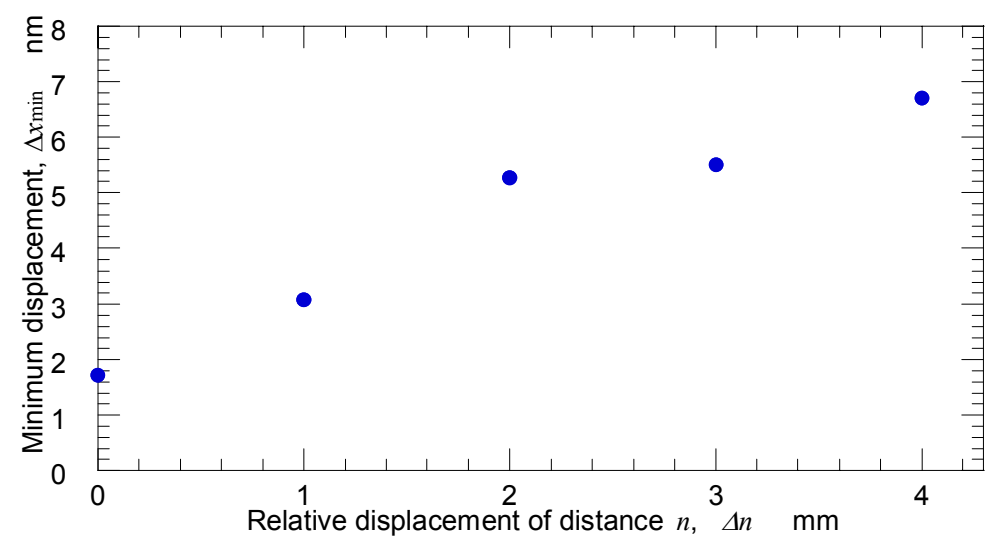

Fig. 13 Effect of distances $\mathrm{n}$ on the detectable minimum displacement.

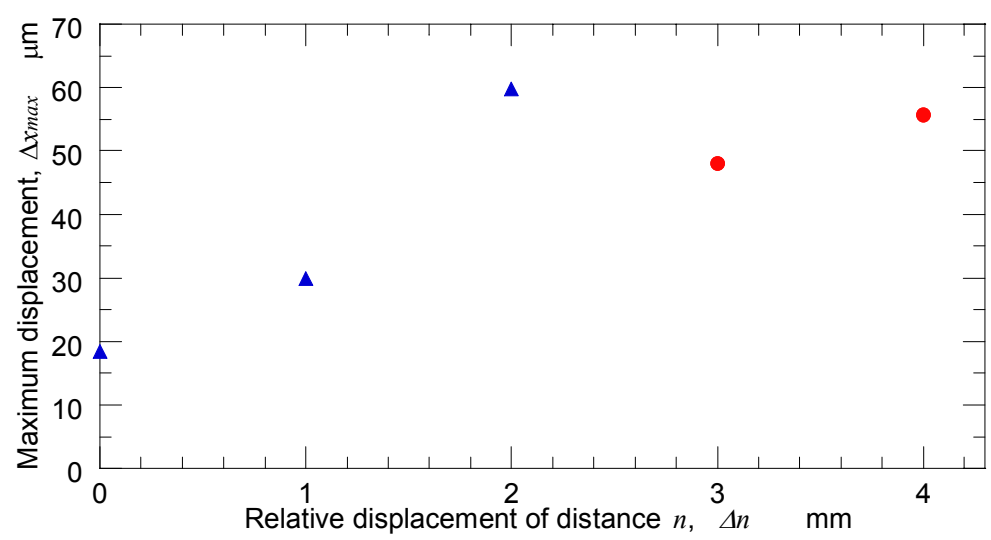

Fig. 14 Effect of distances $\mathrm{n}$ on the detectable maximum displacement.

displacement of distance $n$ was controlled in this experiment, its absolute value was unknown since the position of the focal point of the objective lens could not be precisely determined. Therefore, we plotted the measured results against the relative value of $n$ whose origin indicated the initial position of the probe, which was set at around $n=1-2 \mathrm{~mm}$ by eye. The detectable minimum displacement $\Delta x_{\min }$ was $1.72 \mathrm{~nm}$ at the initial position and it increased with the enlarged distance $n$, which was consistent with the theoretical results shown in Fig. 7.

The experimental results of the detectable maximum displacement $\Delta x_{\max }$ at different $n$ values are plotted in Fig. 14. We evaluated $\Delta x_{\max }$ using the same alignment of the optical system used to measure $\Delta x_{\min }$. Therefore, the transverse axis of Fig. 14 is the same as that in Fig. 13. There are two symbols plotted in Fig. 14: triangles and circles. The data represented by triangles were determined by the probe amplitude just before displacement signal decayed, which was already explained in $\S 3.2$. At $\Delta n=3.0 \mathrm{~mm}$ and $4.0 \mathrm{~mm}$ where the circles are plotted, we did not observe the sudden decay of the signal and could measure the whole resonant curve. This indicated that the width of the laser beam was sufficiently large compared with the probe displacement, which means we could not determine $\Delta x_{\max }$ experimentally. If we could achieve a larger probe amplitude, we might obtain the experimental data of $\Delta x_{\max }$ at these $\Delta n$ values. However, this would require replacing the current piezo actuator with one that could generate a larger driving amplitude, and this was not possible during this study. The circles in Fig. 14 represent the peak values of the resonance curve. In theory, these peak values would not depend on distance $n$ since they were determined by the relationship written in Eq. (1). However, they were slightly 
smaller than $\Delta x_{\max }$ measured at $\Delta n=2.0 \mathrm{~mm}$. This may have been due to the marginal variation of the optical alignment caused by changing the distance $n$. From the triangles in Fig. 14, we confirmed that the $\Delta x_{\max }$ can be increased at the larger distance $n$ as expected from the theoretical results shown in Fig. 6.

Frequency characteristics of our displacement measurement depend on three components of the system, which are the PSD, the signal processor, and the lock-in amplifier. Above these three, the lock-in amplifier has the lowest response. In other words, the frequency characteristics of the measurement are determined only by those of the lock-in amplifier. The lock-in amplifier used can be applied to the synchronous detection ranging from $0.5 \mathrm{~Hz}$ to $100 \mathrm{kHz}$, which sufficiently covers the probe's resonant frequency of around $1-20 \mathrm{kHz}$. The bandwidth whose center is the synchronizing frequency can be set within the range from $5 \mu \mathrm{Hz}$ to $500 \mathrm{~Hz}$. Narrower bandwidth leads to the higher SNR, however, the dynamic response of the measurement is lowered. In the above experiments determining the detectable minimum displacement $\Delta x_{\min }$, the bandwidth was set at $150 \mathrm{~Hz}$ that corresponds to the time constant of around $1 \mathrm{~ms}$. The bandwidth must be adjusted considering the required SNR in the tribological measurement with FWM. Although these frequency characteristics and time responses should be experimentally evaluated with our setup, this was quite difficult since the movement of the shadow in the laser spot on the PSD represents frequency characteristics of both optical fiber probe and the piezo actuator that oscillates the probe. Frequency responses of these mechanical components were much lower than those of the PSD, the signal processor, and the lock-in amplifier. Therefore, it was difficult to achieve the sufficiently-high-frequency oscillation of the shadow required to evaluate the frequency characteristics of the displacement measurement.

The experimental results in Figs. 13 and 14 indicate that we must choose the optimal condition of distance $n$ considering both the range and sensitivity of the displacement measurement required for the high-speed tribological measurement. The detectable maximum amplitude in this study was $59.8 \mu \mathrm{m}$ at $\Delta n=2.0 \mathrm{~mm}$. Since the oscillation frequency when the maximum amplitude was reached was $2.92 \mathrm{kHz}$, the maximum shearing speed achieved was $1.10 \mathrm{~m} / \mathrm{s}$. The minimum detection limit at this shearing speed was 5.26 $\mathrm{nm}$. Considering the shearing conditions in an HDI, a higher shearing speed of $10 \mathrm{~m} / \mathrm{s}$ is required. This will be achieved by using a probe with a higher resonant frequency or setting the experimental setup in a vacuum chamber and increasing the $\mathrm{Q}$ factor of the probe's resonant oscillation. Although we plan to conduct these trials in the future, the displacement measurement method developed in this study is applicable and essential for both cases.

\section{Conclusion}

We developed a novel displacement measurement method that has a wide measuring range of $\pm 100 \mu \mathrm{m}$ and high sensitivity of a few nanometers. This method is essential for high speed tribological measurement using the fiber wobbling method, which can be used to clarify the mechanical properties of monolayer lubricant films designed for lubricating the head disk interface of hard disk drives.

\section{Acknowledgements}

This work was supported in part by SENTAN, the Japan Science Technology Agency (JST), New Energy and Industrial Technology Development Organization, Grant-in-Aid for Scientific Research from the Japan Society for the Promotion of Science (No. 21760111), the Storage Research Consortium, the Electro-mechanic Technology Advancing Foundation, and the Asahi Glass Foundation. 


\section{References}

(1) Itoh, S., Fukuzawa, K., Hamamoto, Y., Zhang, H., and Mitsuya, Y., Fiber wobbling method for dynamic viscoelastic measurement of liquid lubricant confined in molecularly narrow gaps, Tribology Letters, Vol. 30 (2008), pp. 177-189.

(2) Hamamoto, Y., Itoh, S., Fukuzawa, K., and Zhang, H., Nanorheological measurement of monolayer lubricant films using the oscillating optical fiber probe, Proceedings of the STLE/ASME International Joint Tribology Conference, IJTC2008-71138.

(3) Fukuzawa, K., Itoh, S., Ando, T., Takahashi, K., Zhang, H., and Mitsuya, Y., Lateral force measurement using a probe fiber as a microlens, Journal of Applied Physics, Vol. 95, No. 9 (2004), pp. 5189-5191. 